\title{
The Prevalence of Infertility in American Indian/Alaska Natives and Other Racial/Ethnic Groups: National Survey of Family Growth (NSFG)
}

\author{
LaTasha B. Craig ${ }^{1}$, Jennifer D. Peck ${ }^{2}$, and Amanda E. Janitz ${ }^{2}$ \\ 1.Section of Reproductive Endocrinology \& Infertility, Department of Obstetrics and Gynecology, \\ College of Medicine, University of Oklahoma Health Science Center, Oklahoma City, OK \\ 2.Department of Biostatistics and Epidemiology, College of Public Health, University of Oklahoma \\ Health Science Center, Oklahoma City, OK
}

\section{Abstract}

Background: The prevalence of infertility in American Indian/Alaska Native (AI/AN) populations is unknown. The objective of our study was to estimate the prevalence of infertility and impaired fecundity in the $\mathrm{AI} / \mathrm{AN}$ population and other racial and ethnic groups.

Methods: We analyzed female respondent data from the pooled National Survey of Family Growth (NSFG) cycles 2002, 2006-2010 and 2011-2013. We used modified Poisson regression with robust error variance accounting for survey weighting to estimate prevalence proportion ratios (PPR) and 95\% confidence intervals (CI) for NSFG definitions of infertility and impaired fecundity by race and Hispanic ethnicity.

Results: The prevalence of infertility and impaired fecundity in the pooled NSFG was $6.4 \%$ (95\% CI: $5.7 \%, 7.0 \%$ ) and $11.0 \%$ (95\% CI: $11.0 \%, 12.2 \%$ ), respectively. Compared to whites, blacks had a 1.45 times greater adjusted prevalence of infertility $(95 \%$ CI: 1.15, 1.83) and AI/ANs had a 1.37 times greater prevalence of infertility $(95 \%$ CI: $0.91,2.06)$ compared to whites. We observed a 1.30 times greater prevalence of impaired fecundity among AI/AN (95\% CI: 1.04, 1.62) compared to whites. We observed no differences in impaired fecundity for black or Asian/ Native Hawaiian/Pacific Islander women compared to whites or for Hispanic compared to nonHispanic women.

Conclusions: Inequalities in the burden of reproductive impairments among blacks and AI/AN women warrant further evaluation to identify opportunities for prevention and disparity reduction.

\section{Keywords}

Indians; North American; infertility; impaired fecundity; race; ethnicity

Correspondence: Amanda Janitz, PhD, 801 NE $13^{\text {th }}$ St., CHB 309, Oklahoma City, OK, 73104; Phone: 405-271-2229 ×48081; Fax: 405-271-2068; Amanda-Janitz@ ouhsc.edu. 


\section{Background}

Infertility is the inability to conceive within one year of unprotected intercourse and has been identified as a public health priority by the Centers for Disease Control and Prevention (CDC). ${ }^{1}$ The CDC emphasizes that infertility is more than a quality-of-life issue with considerable public health consequences including psychological distress, social stigmatization, economic strain, and marital discord. Furthermore, infertility is associated with increased risk of subsequent chronic health conditions. ${ }^{2-5}$ The public health importance of infertility is reinforced by the World Health Organization ${ }^{6}$ and American Society for Reproductive Medicine, ${ }^{7}$ which define infertility as a reproductive system disease.

A report from the 2006-2010 National Survey of Family Growth (NSFG) estimated that 6\% of married women aged 15-44 years in the U.S. are infertile and $12 \%$ have impaired fecundity, defined as the inability to conceive and carry a baby to term. ${ }^{8}$ Racial disparities in infertility have been observed, with the highest prevalence reported for non-Hispanic black women. ${ }^{8}$ Comparisons of the racial/ethnic burden of infertility, however, have been mostly limited to assessments of blacks, whites, and Hispanics, excluding subgroups such as American Indian/Alaska Native (AI/AN) and Asian/Pacific Islanders (Asian/PI) ${ }^{9,10}$ As a result, the prevalence of infertility in $\mathrm{AI} / \mathrm{AN}$ populations is unknown.

Our objective was to evaluate the prevalence of infertility and impaired fecundity in the $\mathrm{AI} / \mathrm{AN}$ population compared to other racial/ethnic groups to assess potential reproductive health disparities in this underserved population.

\section{Methods}

Study Design.

We used pooled data from the NSFG survey cycles 2002, 2006-2010, and 2011-2013 to examine racial/ethnic variation in infertility prevalence. The NSFG, a national survey using a multistage probability design, is conducted through in-person interviews by the CDC's National Center for Health Statistics including individual participants in each cycle. ${ }^{11,12}$ The pooled NSFG cycles gathered information on infertility and impaired fecundity among men and women ages 15-44 years. We analyzed female respondent data from the pooled NSFG data.

\section{Race and Ethnicity.}

Racial groups were categorized by NSFG as 1) AI/AN; 2) Asian/PI; 3) black; or 4) white. NSFG respondents self-reported their racial group and were allowed to choose up to four races. Respondents reporting multiple races were asked to choose the racial group that best described them, which we used in our analyses. Ethnicity was categorized as Hispanic or Non-Hispanic (NH).

\section{Outcome Measures of Infertility and Impaired Fecundity.}

We examined differences in the prevalence of infertility and impaired fecundity by race/ ethnic group. The prevalence of infertility, a couple-based measure, was our primary outcome of interest. Consistent with NSFG definitions, ${ }^{8}$ infertility was defined as " 12 or 
more months of intercourse without pregnancy and without contraception" among women who were married or cohabiting. Women in this analysis were also classified as surgically sterile or presumed fertile (residual of women who did not meet the definition of infertile or surgically sterile, but are married/cohabiting) and were included in the denominator, which is consistent with previous studies. ${ }^{8,9}$ For respondents with missing infertility data, the NSFG used regression imputation $(n=13)$ and logical imputation conducted by subjectmatter experts at the National Center for Health Statistics $(n=49)$ to impute responses.

Impaired fecundity was defined as all women, regardless of marital or cohabiting status, who (i) reported it was not physically possible to conceive (excluding surgical sterilization); (ii) were classified as subfecund (i.e., difficult for a couple to conceive/deliver a baby or received medical advice to not become pregnant); or (iii) had a long interval without conception (i.e., no pregnancy in the 36 months prior to interview when married/cohabiting continuously, without contraception, and no months without intercourse). Women were classified as fecund if the respondent was currently pregnant and her husband/partner was not surgically sterile or she did not meet the criteria for one of the other impaired fecundity categories. Women with impaired fecundity or who were surgically sterile were included in the denominator for consistency with prior reporting. ${ }^{8}$ The respondent was classified as surgically sterile if this was reported during the interview. All respondents provided a response to the question on impaired fecundity with no imputation conducted.

\section{Covariates.}

We obtained demographic characteristics from the NSFG, which included age at interview, marital status (currently married to a person of the opposite sex, not married but living with opposite sex partner, widowed, divorced or annulled, separated for reasons of marital discord, never been married), education (years), poverty level ( $<150,150-299$, and $\geq 300$ percent of the Federal Poverty Level of the survey year), parity (number of live births), body mass index (BMI), smoking during the last three months (none, $<1 / 2$ pack per day, $\geq 1 / 2$ pack per day), pelvic inflammatory disease treatment (ever/never), use of Depo-Provera (ever/never), age at menarche (years), any medical help to become pregnant (ever/never), total family income of the respondent in the calendar year before the interview $(<\$ 14,999$, $\$ 15,000-\$ 34,999, \$ 35,000-\$ 59,999, \$ 60,000)$, and gynecologic disorders (ever/never diagnosed with fibroids, endometriosis, or ovulation problems).

\section{Statistical Methods.}

All data analyses were conducted via remote access to the NCHS Research Data Center (RDC) using SAS v. 9.4. Initial analyses evaluated weighted counts, percentages, and 95\% confidence intervals by infertility/impaired fecundity status. We used modified Poisson regression with robust error variance to assess the relationship between race and ethnicity and the prevalence of 1 ) infertility and 2) impaired fecundity accounting for the complex survey design to estimate prevalence proportion ratios (PPR). Covariates evaluated in the multivariable regression models included age, parity, marital status, education, poverty level, body mass index, smoking, pelvic inflammatory disease treatment, ever use of DepoProvera, age at menarche, any medical help to become pregnant, income, and gynecologic disorders. For comparison with previous findings, ${ }^{8}$ we also evaluated models adjusted for 
age, parity, marital status, education, and poverty level only. This study was reviewed by the University of Oklahoma Health Sciences Center Institutional Review Board and classified as exempt.

\section{Results}

The prevalence of infertility and impaired fecundity was 6.4\% (95\% CI: 5.7\%, 7.0\%) and $11.0 \%$ (95\% CI: $11.0 \%, 12.2 \%$ ), respectively. When evaluating descriptive characteristics of women who were infertile or had impaired fecundity, many demographic, behavioral, and reproductive factors differed by infertility and fecundity status (Table 1). Respondents with infertility were more often black (13\%) compared to those who were fecund/surgically sterile (10\%); approximately $5 \%$ of women in each group were AI/AN. Similar proportions $(16 \%)$ of women with and without impaired fecundity were black; AI/AN women made up $6 \%$ and $5 \%$ of women with and without impaired fecundity, respectively.

Compared to whites, blacks had a 1.45 times greater adjusted prevalence of infertility ( $95 \%$ CI: $1.15,1.83$ ) (Table 2). AI/ANs had a 1.37 times greater prevalence of infertility compared to whites (95\% CI: $0.91,2.06$ ), though the $95 \%$ confidence interval included the reference value of 1.0. The adjusted PPR for infertility among Asian/PI women compared to white women (PPR: 0.89, 95\% CI: 0.53-1.49) was not increased. The adjusted PPR for Hispanic women compared to non-Hispanic women was 1.23 (95\% CI: 0.95-1.58). In analysis of impaired fecundity, we observed a 1.30 times greater adjusted prevalence among AI/AN women (95\% CI: 1.04, 1.62) compared to whites (Table 3). No increased PPRs were observed for black and Asian/PI women compared to whites, or among Hispanic compared to non-Hispanic women.

\section{Comment}

We observed increased infertility among black women, and increased infertility and impaired fecundity among AI/AN women, relative to whites. No differences in prevalence of infertility or impaired fecundity were observed for other racial groups compared to whites or by Hispanic ethnicity. Our results demonstrating increased infertility and impaired fecundity among black women compared to white women were consistent with both 2002 (adjusted OR: $1.98,95 \%$ CI: $1.28,3.06$ ) and 2006-2010 (adjusted OR: 1.84, 95\% CI: 1.10, 3.06) NSFG estimates, indicating consistency over time ${ }^{8,9}$ Our results were also consistent with a prospective study, which observed an association with infertility among black compared to white women, excluding surgically sterile women (adjusted OR: 1.97, 95\% CI: 1.25, 3.12).

${ }^{10}$ No other studies have evaluated the prevalence of infertility and impaired fecundity among AI/AN women. Disparities in infertility are likely due to differential distributions of factors such as age, education, socioeconomic status, health behaviors, acute and chronic health conditions, exposure to environmental and infectious agents, access to quality infertility services, and service-seeking behaviors, though research is limited. ${ }^{1}$

A strength was the ability to evaluate infertility and impaired fecundity over several NSFG cycles. One limitation is the classification of infertility and impaired fecundity. Measures of infertility were self-reported and do not equate with medical evaluation and treatment. 
NSFG participants were not queried specifically about infertility. Instead, it was constructed from survey questions addressing marital status, sexual activity, and contraceptive use. In addition, the NSFG definition of infertility was restricted to married or cohabiting women ages 15-44 years who have not used contraception in the previous 12 months, which limits the generalizability of the estimates.

Inequalities in the burden of reproductive impairments among blacks and AI/AN women warrant further evaluation of the contribution of underlying causes of infertility/impaired fecundity and opportunities to reduce disparities. Given mounting evidence of maternal/child health disparities among AI/ANs, ${ }^{13-15}$ it is imperative to understand the contribution of infertility to the reproductive health status of this underserved population.

\section{Acknowledgements:}

This project is supported by the Health Resources and Services Administration (HRSA) of the U.S. Department of Health and Human Services (HHS) under 1 R40MC29449-01-00 and by Oklahoma Shared Clinical and Translational Resource Institute NIGMS U54 GM104938. The information, content and/or conclusions are those of the authors and should not be construed as the official position or policy of, nor should any endorsements be inferred by HRSA, HHS or the U.S. The findings and conclusions in this paper are those of the author(s) and do not necessarily represent the views of the Research Data Center, the National Center for Health Statistics, or the Centers for Disease Control and Prevention.

\section{References}

1. Centers for Disease Control and Prevention. National Public Health Action Plan for the Detection, Prevention, and Management of Infertility. Atlanta, GA: Centers for Disease Control and Prevention;2014.

2. Eisenberg ML, Li S, Behr B, Pera RR, Cullen MR. Relationship between semen production and medical comorbidity. Fertil Steril. 2015;103(1):66-71. [PubMed: 25497466]

3. Kurabayashi T, Mizunuma H, Kubota T, Hayashi K. Ovarian infertility is associated with cardiovascular disease risk factors in later life: A Japanese cross-sectional study. Maturitas. 2015.

4. Meczekalski B, Katulski K, Czyzyk A, Podfigurna-Stopa A, Maciejewska-Jeske M. Functional hypothalamic amenorrhea and its influence on women's health. Journal of endocrinological investigation. 2014;37(11):1049-1056. [PubMed: 25201001]

5. Tobias DK, Gaskins AJ, Missmer SA, et al. History of infertility and risk of type 2 diabetes mellitus: a prospective cohort study. Diabetologia. 2015;58(4):707-715. [PubMed: 25596853]

6. Zegers-Hochschild F, Adamson GD, de Mouzon J, et al. International Committee for Monitoring Assisted Reproductive Technology (ICMART) and the World Health Organization (WHO) revised glossary of ART terminology, 2009. Fertil Steril. 2009;92(5):1520-1524. [PubMed: 19828144]

7. Practice Committee of American Society for Reproductive Medicine. Definitions of infertility and recurrent pregnancy loss: a committee opinion. Fertil Steril. 2013;99(1):63. [PubMed: 23095139]

8. Chandra A, Copen CE, Stephen EH. Infertility and impaired fecundity in the United States, 19822010: data from the National Survey of Family Growth. National health statistics reports. 2013(67): $1-18$.

9. Thoma ME, McLain AC, Louis JF, et al. Prevalence of infertility in the United States as estimated by the current duration approach and a traditional constructed approach. Fertil Steril. 2013;99(5): 1324-1331 e1321. [PubMed: 23290741]

10. Wellons MF, Lewis CE, Schwartz SM, et al. Racial differences in self-reported infertility and risk factors for infertility in a cohort of black and white women: the CARDIA Women's Study. Fertil Steril. 2008;90(5):1640-1648. [PubMed: 18321499]

11. Lepkowski JM, Mosher MJ, Davis KE, Groves RM, van Hoewyk J, Willem J. National Survey of Family Growth, Cycle 6: Sample design, weighting, imputation, and variance estimation. Vital Health Stat. 2006;2(142):1-82. 
12. Lepkowski JM, Mosher WD, Davis KE, Groves RM, Van Hoewyk J. The 2006-2010 National Survey of Family Growth: Sample design and analysis of a continuous survey. Vital Health Stat. 2010;2(150):1-36.

13. Wong CA, Gachupin FC, Holman RC, et al. American Indian and Alaska Native Infant and Pediatric Mortality, United States, 1999-2009. American Journal of Public Health. 2014:e1-e9.

14. Craig LB, Weedin EA, Walker WD, Janitz AE, Hansen KR, Peck JD. Racial and Ethnic Differences in Pregnancy Rates Following Intrauterine Insemination with a Focus on American Indians. Racial and Ethnic Health Disparities 2018;

15. Kreisel K, Torrone E, Bernstein K, Hong J, Gorwitz R. Prevalence of Pelvic Inflammatory Disease in Sexually Experienced Women of Reproductive Age - United States, 2013-2014. MMWR Morb Mortal Wkly Rep. 2017;66(3):80-83. [PubMed: 28125569] 


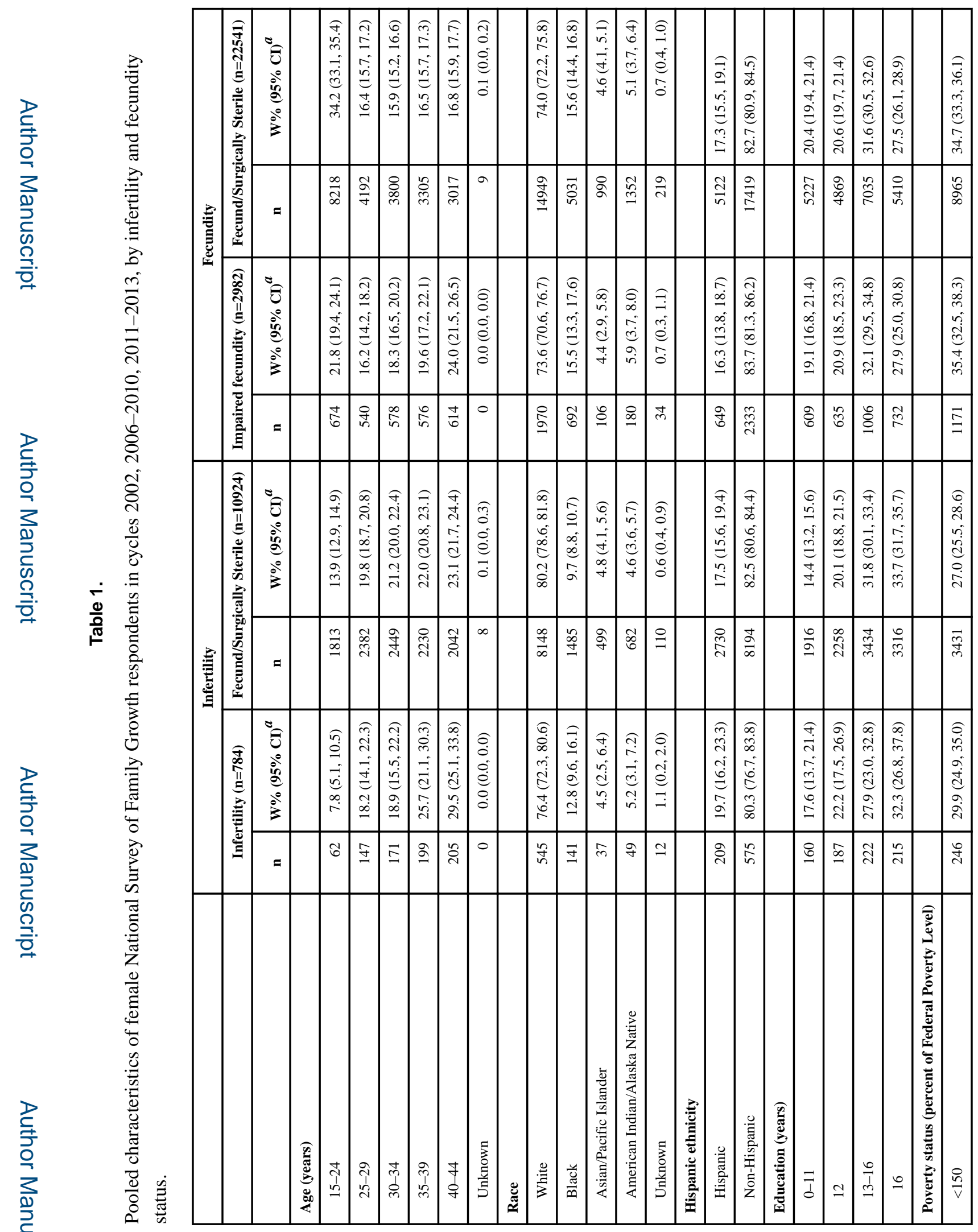

Paediatr Perinat Epidemiol. Author manuscript; available in PMC 2020 March 01. 


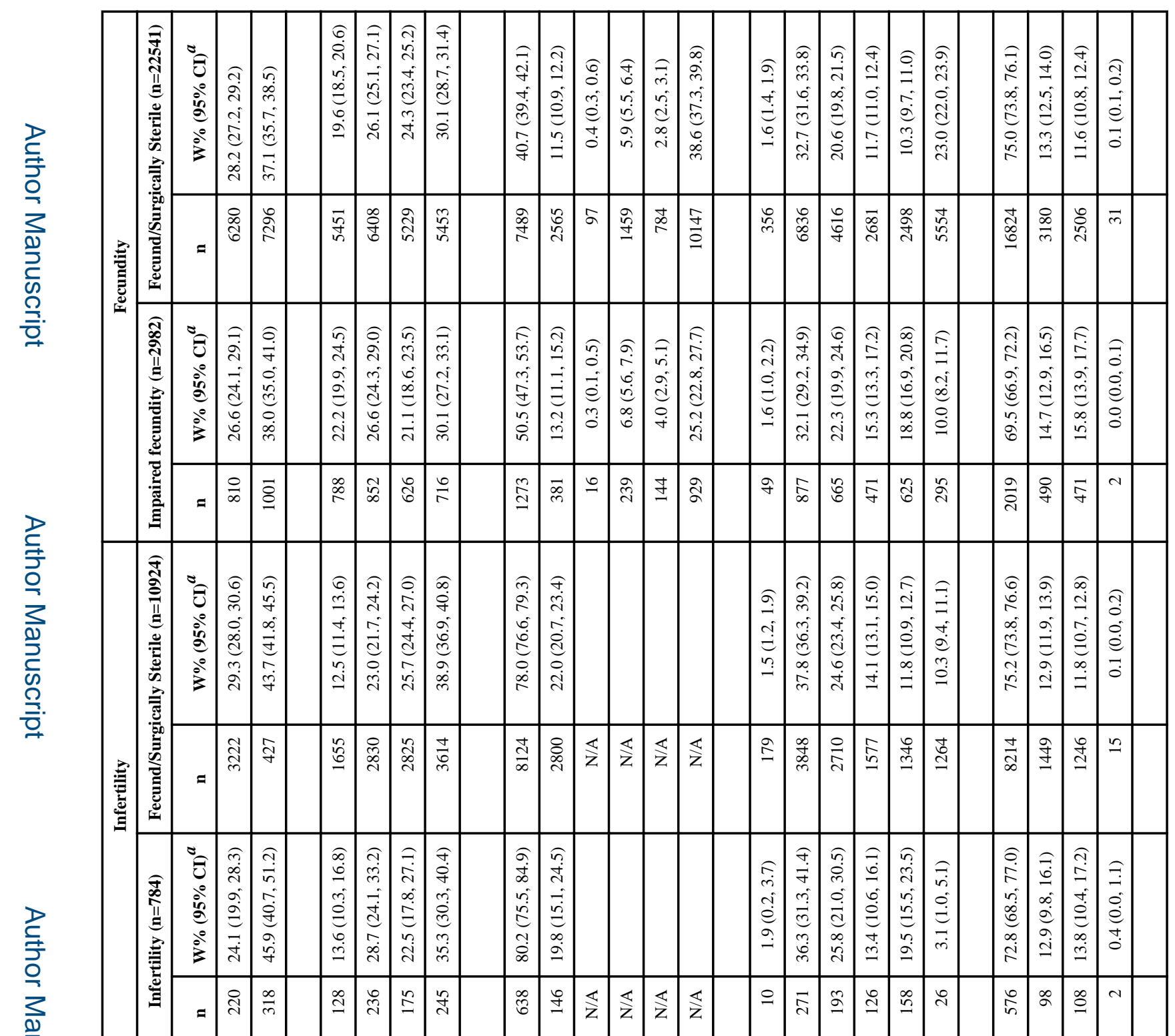

을

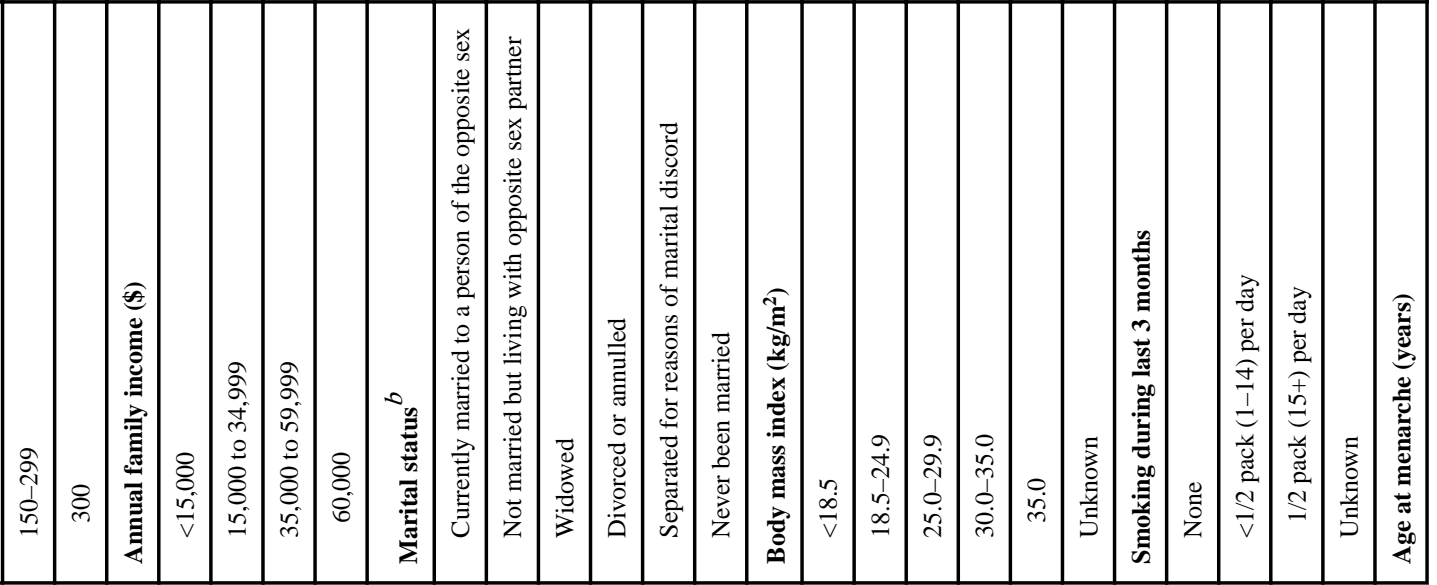

Paediatr Perinat Epidemiol. Author manuscript; available in PMC 2020 March 01. 


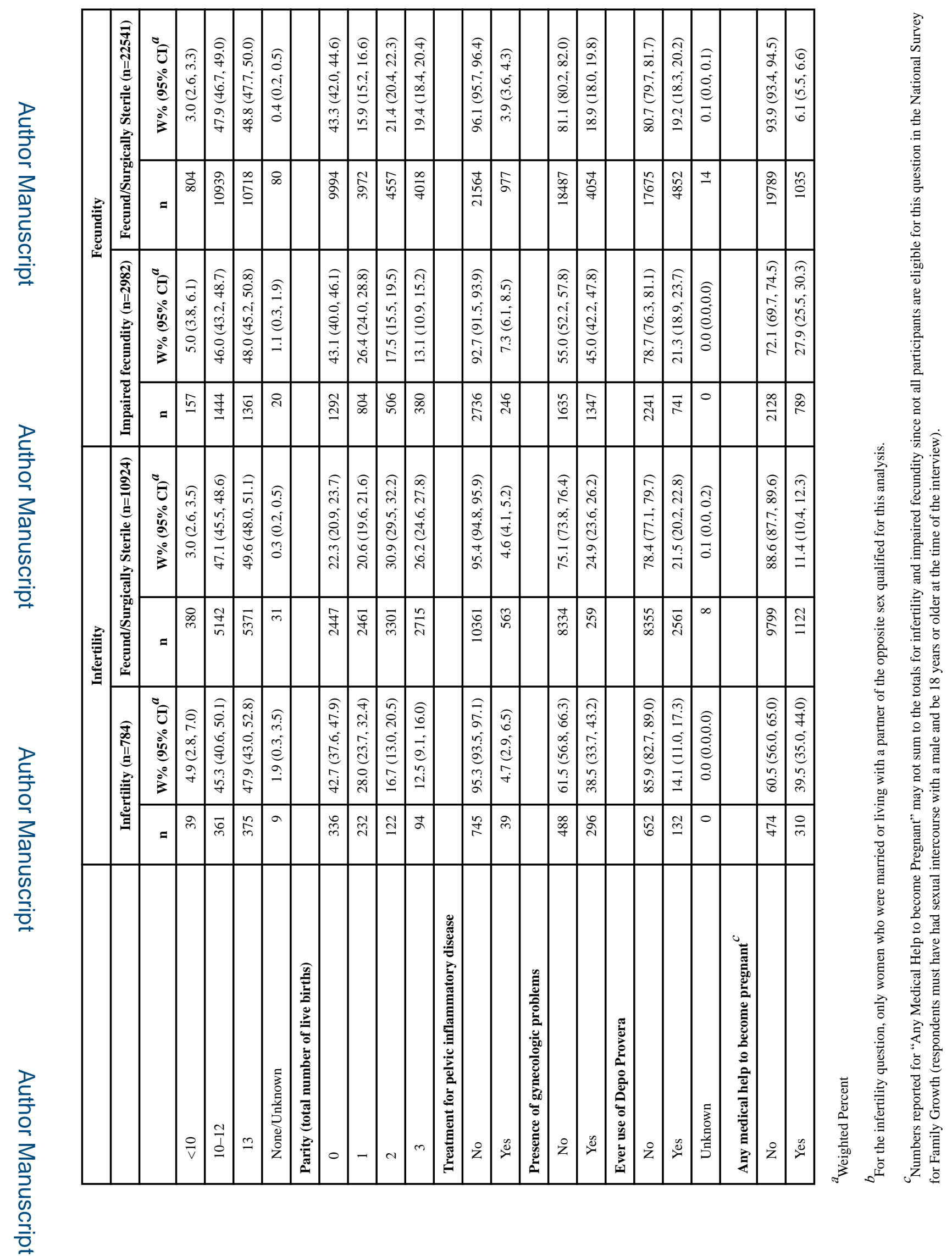

Paediatr Perinat Epidemiol. Author manuscript; available in PMC 2020 March 01. 


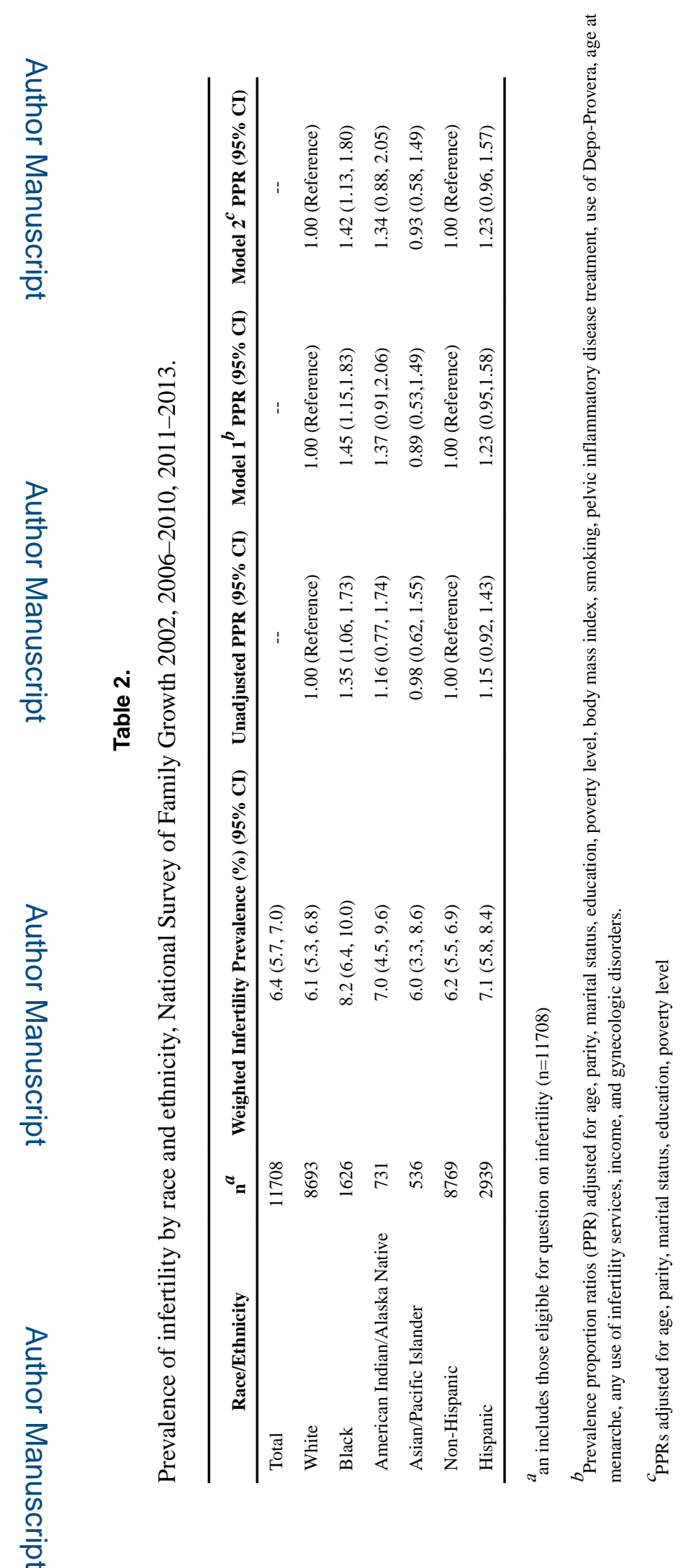

Paediatr Perinat Epidemiol. Author manuscript; available in PMC 2020 March 01. 


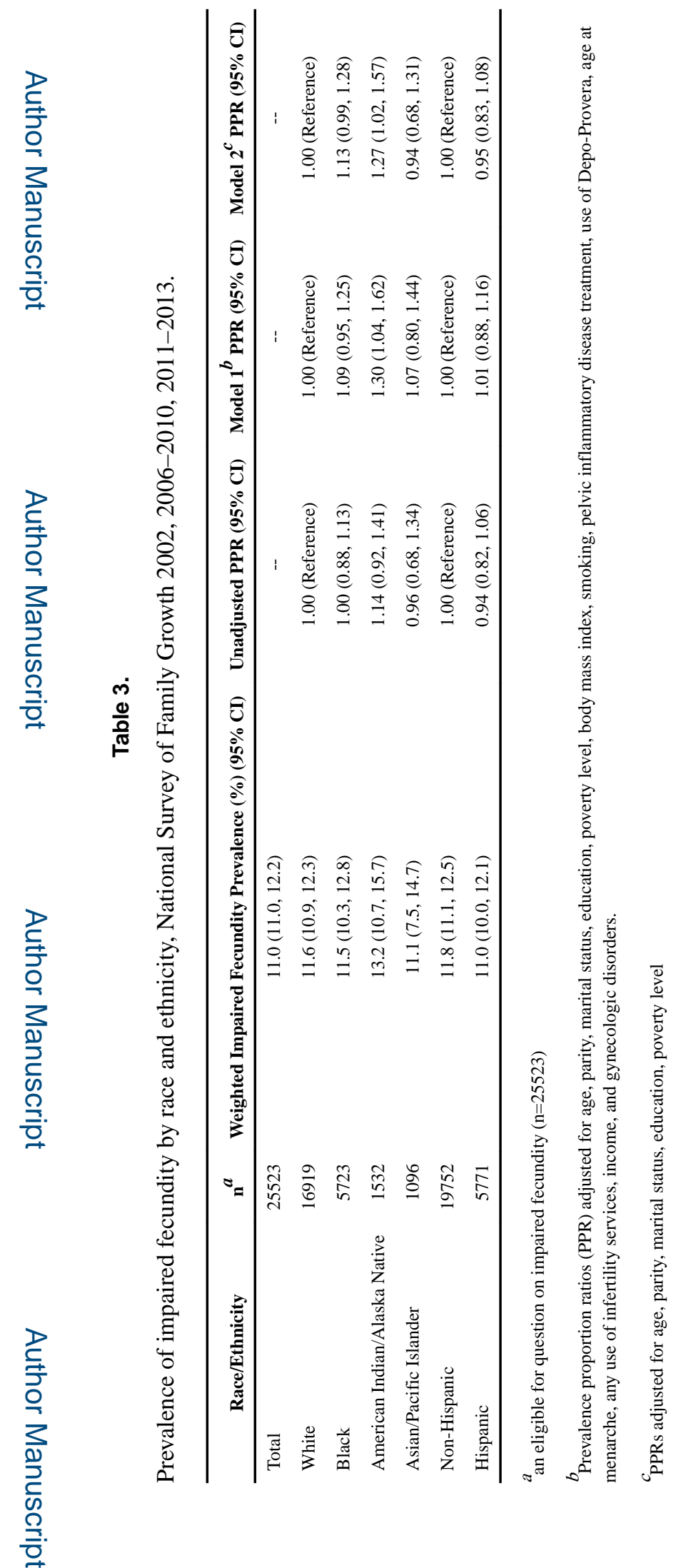

Paediatr Perinat Epidemiol. Author manuscript; available in PMC 2020 March 01. 